\title{
Spin-dependent Klein tunneling in graphene: Role of Rashba spin-orbit coupling
}

\author{
Ming-Hao Liu (劉明豪), Jan Bundesmann, and Klaus Richter \\ Institut für Theoretische Physik, Universität Regensburg, D-93040 Regensburg, Germany \\ (Received 2 December 2011; revised manuscript received 12 January 2012; published 3 February 2012)
}

\begin{abstract}
Within an effective Dirac theory the low-energy dispersions of monolayer graphene in the presence of Rashba spin-orbit coupling and spin-degenerate bilayer graphene are described by formally identical expressions. We explore implications of this correspondence for transport by choosing chiral tunneling through pn and pnp junctions as a concrete example. A real-space Green's function formalism based on a tight-binding model is adopted to perform the ballistic transport calculations, which cover and confirm previous theoretical results based on the Dirac theory. Chiral tunneling in monolayer graphene in the presence of Rashba coupling is shown to indeed behave like in bilayer graphene. Combined effects of a forbidden normal transmission and spin separation are observed within the single-band $n \leftrightarrow p$ transmission regime. The former comes from real-spin conservation, in analogy with pseudospin conservation in bilayer graphene, while the latter arises from the intrinsic spin-Hall mechanism of the Rashba coupling.
\end{abstract}

DOI: 10.1103/PhysRevB.85.085406

PACS number(s): 72.80.Vp, 72.25.-b, 73.23.-b, 73.40.Gk

\section{INTRODUCTION}

After the first successful isolation of monolayer graphene (MLG) was announced, ${ }^{1}$ intriguing properties based on its low-energy excitation that mimics massless, gapless, and chiral Dirac fermions were intensively investigated. ${ }^{2,3}$ Spin-orbit coupling (SOC), on the other hand, is the key ingredient of semiconductor spintronics ${ }^{4,5}$ that was undergoing a rapid development before the rise of graphene. ${ }^{6}$ The question about the role of SOC effects in graphene then naturally emerged, including the proposal of graphene as a topological insulator, ${ }^{7}$ which attracted the attention of various first-principles-based studies. $^{8-10}$

SOC in MLG includes an intrinsic and an extrinsic term. The former reflects the inherent asymmetry of electron hopping between next nearest neighbors ${ }^{7}$ (i.e., a generalization of Haldane's model ${ }^{11}$ ). The latter is induced by the electric field perpendicular to the graphene plane, which can be externally controlled, and resembles the Rashba model ${ }^{12,13}$ for the two-dimensional electron gas. Agreement has been achieved, based on first-principles calculations, ${ }^{9,10}$ that the intrinsic SOC term opens a gap of the order of $2 \lambda_{I} \approx 24 \mu \mathrm{eV}$, while the Rashba SOC removes the spin degeneracy and creates a spin-splitting $2 \lambda_{R}$ at the $K$ and $K^{\prime}$ points that has a linear dependence on an external electric field $E$ with the slope of about $100 \mu \mathrm{eV}$ per $\mathrm{V} / \AA$ of $E$. Under a strong gate voltage, the Rashba coupling may in principle dominate the intrinsic SOC in MLG. ${ }^{9,10}$

The low-energy spectrum of MLG plus the Rashba coupling $(\mathrm{MLG}+\mathrm{R})$ was derived by Rashba,${ }^{14}$ based on the Kane-Mele model $^{7}$ (i.e., an effective Dirac Hamiltonian). An earlier work by one of us ${ }^{15}$ started with a tight-binding model (TBM) and obtained an equivalent form of the low-energy expansion, ${ }^{16}$

$$
E_{\mathrm{MLG}+\mathrm{R}}(\mathbf{q}) \approx \mu \frac{1}{2}\left[\sqrt{\left(3 t_{R}\right)^{2}+(3 t a \cdot q)^{2}}+v\left(3 t_{R}\right)\right]
$$

which also agrees with expressions given in Refs. 9 and 10 when $\lambda_{I}=0$. Here $\mu, v= \pm 1$ are band indices, $t$ and $t_{R}$ are nearest-neighbor kinetic and Rashba hopping parameters, respectively, $a \approx 1.42 \AA$ is the bonding length, and $\mathbf{q}=K+\delta \mathbf{k}$ with $|\delta \mathbf{k}| a \ll 1$. Recall for comparison the low-energy spectrum of bilayer graphene (BLG), ${ }^{2,17}$

$$
E_{\mathrm{BLG}}(\mathbf{q}) \approx \mu \frac{1}{2}\left(\sqrt{\gamma_{1}^{2}+(3 t a \cdot q)^{2}}+v \gamma_{1}\right),
$$

where $\gamma_{1}$ is the nearest-neighbor hopping between the two graphene layers. Note that the next nearest-neighbor interlayer hoppings $\gamma_{3}$ and $\gamma_{4}$ do not influence the band dispersion near $K$. The completely different mechanisms of (i) pseudospin coupling between carriers from the two graphene layers of BLG through interlayer hopping $\gamma_{1}$ and (ii) real-spin coupling between up and down spins within MLG through Rashba hopping $t_{R}$ happen to lead to an identical mathematical form in Eqs. (1) and (2) that can be clearly mapped onto each other ${ }^{18,19}$ with $\gamma_{1} \leftrightarrow 3 t_{R}$ as sketched in Fig. 1. This unambiguously implies that low-energy physics in MLG $+\mathrm{R}$ and BLG should behave similarly.

In this paper we tackle the question of whether the transport in $\mathrm{MLG}+\mathrm{R}$ behaves as in BLG by choosing the issue of

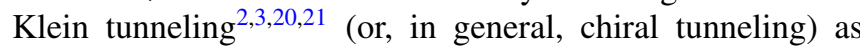
a concrete example. Chiral tunneling in graphene has been shown to exhibit completely different behavior in MLG and BLG based on the Dirac theory. ${ }^{22}$ Tunneling at normal incidence in MLG shows a suppression of backscattering, which resembles the original Klein paradox in relativistic quantum electrodynamics ${ }^{23}$ and hence the name Klein tunneling, while in BLG it shows a perfect reflection, which is strictly speaking a consequence of forbidden interband transition also due to the

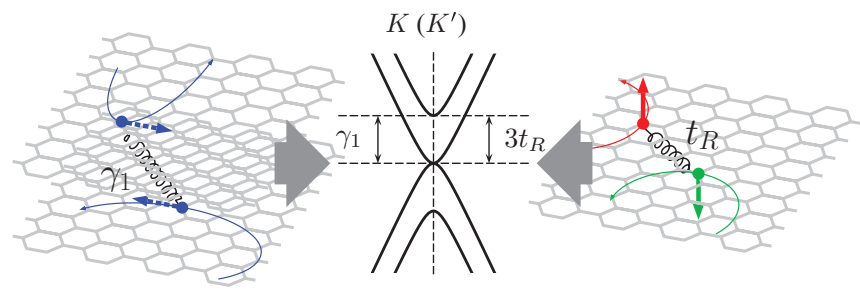

FIG. 1. (Color online) Schematic of the pseudospin coupling through $\gamma_{1}$ in BLG (left panel) and real-spin coupling through $t_{R}$ in MLG (right), which lead to an identical low-energy dispersion near $K$ and $K^{\prime}$. 
chiral nature of graphene. The theoretical discussion of chiral tunneling so far focuses mainly on spin-independent tunneling through $p n$ and $p n p$ junctions, ${ }^{21,22,24-30}$ while SOC effects are less discussed. ${ }^{18,31-33}$ In addition, the relevant theoretical understanding so far is based on Dirac theory, which is valid only for the Fermi level close to the charge neutrality point and allows one only to consider certain relatively simple systems. A recent study discussing the interplay between the AharanovBohm effect and Klein tunneling in graphene, started with a TBM,${ }^{34}$ but the nanoribbon type of the leads used in that work may have edge effects included that can be very different from the bulk properties of graphene. A more transparent theoretical study of chiral tunneling in graphene directly bridging the analytical Dirac theory and the numerical TBM computation is so far missing and deserves consideration.

In the present work, we re-treat this issue of chiral tunneling in graphene based on the TBM and show a unified description, allowing for a broad range of geometries and complementing the existing results based on the Dirac theory. Straightforward generalization to the case of MLG + R reveals a spin-dependent tunneling behavior in close analogy with that in BLG, with the role of pseudospin in BLG replaced by real spin in MLG + R. Specifically, a combined behavior of spin-Hall-based spin separation and suppression of normal transmission will be shown.

This paper is organized as follows. In Sec. II we briefly summarize the theoretical formalism applied in the present calculation, namely, real-space Green's function formalism in noninteracting bulk graphene. In Sec. III we show our TBM results including the consistency with the Dirac theory, a direct comparison between BLG and $\mathrm{MLG}+\mathrm{R}$, and a deeper discussion of the MLG $+\mathrm{R}$ case. We review also briefly the recent experimental progress on the Rashba spin splitting and Klein tunneling in graphene in Sec. IV, and finally conclude in Sec. V.

\section{FORMALISM}

\section{A. Tight-binding model for "bulk" graphene}

We choose the TBM for describing the electronic properties of graphene, which is a well established way to treat graphene numerically. For spin-degenerate MLG, the Hamiltonian reads

$$
\mathcal{H}_{\mathrm{MLG}}=\sum_{i} V_{i} c_{i}^{\dagger} c_{i}-t \sum_{\langle i, j\rangle} c_{i}^{\dagger} c_{j}
$$

where the operator $c_{i}^{\dagger}\left(c_{i}\right)$ creates (annihilates) an electron at site $i$ (including both sublattices $A$ and $B$ ). The first sum in Eq. (3) runs over all the atomic sites in the considered region with on-site potential $V_{i}$, and the second sum runs over all the pairs of neighboring atomic orbitals $\langle i, j\rangle$ with kinetic hopping parameter $t(\approx 3 \mathrm{eV})$. The next nearest neighbor kinetic hopping term, usually characterized by $t^{\prime} \approx 0.1 t$, can be added in Eq. (3) but will not be considered in the present work due to the minor role it plays in the bulk transport properties for low-energy excitation.
Spin-orbit interactions can be incorporated into the TBM by altering the spin-dependent hopping between nearest and next-nearest neighbors, ${ }^{7,35}$ modifying Eq. (3) as

$$
\mathcal{H}_{\mathrm{MLG}+\mathrm{R}}=\sum_{i} V_{i} \sigma^{0} c_{i}^{\dagger} c_{i}+\sum_{\langle i, j\rangle} c_{i}^{\dagger}\left[-t \sigma^{0}+i t_{R}\left(\vec{\sigma} \times \mathbf{d}_{i j}\right)_{z}\right] c_{j} .
$$

Here $\sigma^{0}$ is the $2 \times 2$ identity matrix, $t_{R}$ is the Rashba spin-orbit hopping parameter, $\mathbf{d}_{i j}$ is the unit vector pointing from site $j$ to $i$, and $\vec{\sigma}=\left(\sigma^{x}, \sigma^{y}, \sigma^{z}\right)$ is the vector of (real-) spin Pauli matrices. We take into account only the extrinsic SOC and neglect the intrinsic term in order to highlight the role of the Rashba SOC.

For spin-degenerate BLG, we consider

$$
\mathcal{H}_{\mathrm{BLG}}=\sum_{m=1,2} \mathcal{H}_{\mathrm{MLG}}^{(m)}-\gamma_{1} \sum_{j}\left(b_{2, j}^{\dagger} a_{1, j}+\text { H.c. }\right),
$$

where $\mathcal{H}_{\mathrm{MLG}}^{(m)}$ is $\mathcal{H}_{\mathrm{MLG}}$ given by Eq. (3) of the $m$ th graphene layer, $a_{m, j}\left(b_{m, j}\right)$ annihilates an electron on sublattice $A$ $(B)$ in layer $m=1,2$ at unit cell $j$ (that contains two sublattice sites belonging to $A$ and $B$ ), and the interlayer coupling strength $\gamma_{1} \approx 0.4 \mathrm{eV}$ corresponds to the nearest neighbor hopping between the two MLG layers. Further interlayer hopping terms, ${ }^{2}-\gamma_{4} \sum_{j}\left(a_{2, j}^{\dagger} a_{1, j}+b_{2, j}^{\dagger} b_{1, j}+\right.$ H.c. $)$ and $-\gamma_{3} \sum_{j}\left(a_{2, j}^{\dagger} b_{1, j}+\right.$ H.c. $)$, are not considered in the present calculation, since they do not influence the low-energy excitation. Throughout the presentation of the numerical results in Sec. III, the kinetic hopping parameters will be fixed at $t=3 \mathrm{eV}$ and $\gamma_{1}=0.39 \mathrm{eV}$, while the value of the Rashba hopping parameter $t_{R}$ depends on the context.

For the simulation of bulk graphene, we impose the Bloch theorem along the transverse direction with periodicity $W$. This is equivalent to considering a nanoribbon and modifying the hopping between atomic sites connected through the periodic boundary conditions by a Bloch phase factor $e^{i k_{B} W}$ with a Bloch momentum $k_{B},{ }^{36}$ as schematically shown for MLG in Fig. 2. At the same time the Bloch momentum is the component of the electron's momentum perpendicular to the nanoribbon, hence defining the propagation angle $\phi=\sin ^{-1}\left(k_{B} / k_{F}\right)$, where $k_{F}$ is the Fermi wave vector. To be consistent with the literature related to Klein tunneling based on the Dirac theory, in Sec. III we will refer to the Bloch momentum as $k_{y}$.

In the present calculations, we will apply a minimal TBM by imposing the periodic boundary conditions on a zigzag nanoribbon with chain number $N_{z}=2$, that is, periodicity of $W=3 a$ (as the case sketched in Fig. 2). The present model applies equally well for metallic armchair ribbon (chain

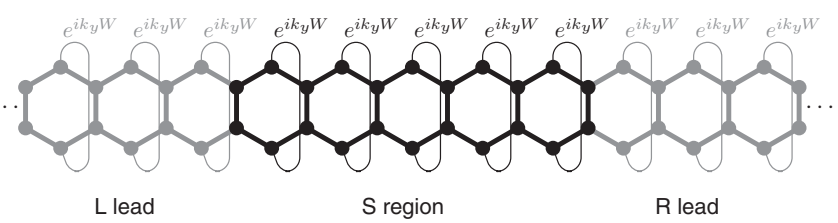

FIG. 2. Schematic of a minimum tight-binding model that simulates a bulk MLG up to nearest neighbor hoppings with $W=3 a$. Further nearest neighbor hoppings can be accounted for by enlarging the transverse periodicity $W$ to at least $6 a$. 
number $N_{a}$ being a multiple of 3 ) with periodic boundary conditions, but the minimal model would require $N_{a}=3$ (i.e., periodicity of $W=3 \sqrt{3} a$ ).

\section{B. Brief summary of real-space Green's function formalism}

We consider open systems connected to the outer world by two leads (see Fig. 2). According to the real-space Green's function formalism ${ }^{37}$ we numerically calculate the Green's functions of our system,

$$
G_{S}^{r / a}=\left[E-H_{S}-\Sigma^{r / a} \pm i \eta\right]^{-1},
$$

where the self-energies of the leads $\left(\Sigma^{r / a}=\Sigma_{L}^{r / a}+\Sigma_{R}^{r / a}\right)$ reflect the fact that our system is open. The powerful recipe constructed in Ref. 36 for graphene handles a lead as a semiinfinite repetition of unit cells and allows for incorporating any kind of lattice structure and one-body interaction such as SOCs. The transmission probability for an electron traveling from lead $L$ to lead $R$ is given by the Fisher-Lee relation, ${ }^{36,37}$

$$
T_{R L}=\operatorname{Tr}\left(\Gamma_{L} G_{S}^{r} \Gamma_{R} F_{S}^{a}\right),
$$

where the trace is done with respect to the lattice sites. The spectral matrix functions $\Gamma_{L / R}$ are given by the lead self-energies as $\Gamma_{L / R}=i\left(\Sigma_{L / R}^{r}-\Sigma_{L / R}^{a}\right)$.

For a given Bloch momentum $k_{y}$ and a given Fermi energy $E_{F}$ [subject to a Fermi wave vector $k_{F}$ via Eq. (1) for $\mathrm{MLG}+\mathrm{R}$ or Eq. (2) for BLG], the incoming propagation angle $\phi$ of the electron wave can be defined as $\phi=\sin ^{-1}\left(k_{y} / k_{F}\right)$. The angle-dependent transmission function $T(\phi)$ is obtained from Eq. (7), which can be generalized to a spin-resolved version. ${ }^{38}$

\section{TRANSPORT RESULTS}

In this section we present numerical results of our tightbinding transport calculations. We first show the consistency of our tight-binding calculations with the existing effective Dirac theory in Sec. III A. A direct comparison between BLG and $\mathrm{MLG}+\mathrm{R}$ will then be shown in Sec. III B. Finally, Sec. III C is devoted to MLG $+\mathrm{R}$ for $p n$ junctions, in particular the role of Rashba SOC for chiral tunneling.

\section{A. Consistency with Dirac theory}

We first consider tunneling in graphene without SOC and confirm existing results, limited to low-energy excitations, by our tight-binding calculations. We pick two pioneering theoretical works to demonstrate the consistency explicitly. Consistency with recent works of tunneling in graphene heterojunctions in the presence of $\mathrm{SOC}^{18,31}$ has also been checked, but is not explicitly shown here.

\section{Chiral tunneling in $M L G$ vs $B L G$}

Tunneling in MLG and BLG behaves quite differently as mentioned in Sec. I and pointed out by Katsnelson et al. ${ }^{22}$ For a quantitative comparison we consider a barrier of width $D=100 \mathrm{~nm}$ and the incoming Fermi wave vector $k_{F}=$ $2 \pi / 50 \mathrm{~nm}^{-1}$ as in Ref. 22 for both MLG and BLG [see Figs. 3(a) and 3(c)]. Note that in order to exactly match the barrier width, we set the bonding length $a=(4 \sqrt{3})^{-1} \mathrm{~nm}$, which differs from the realistic value of about $1.42 \AA$ by

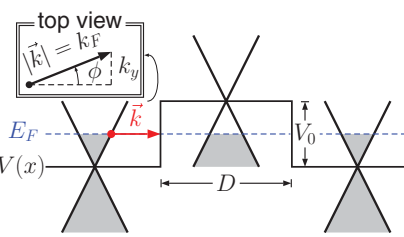

(a)

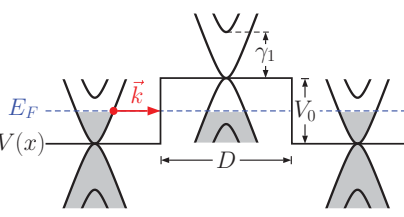

(c)

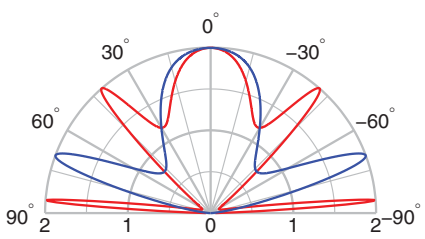

(b)

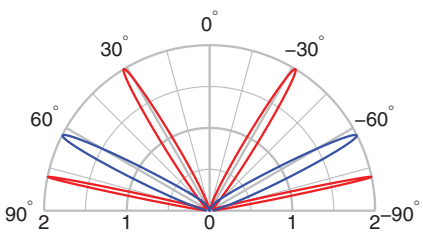

(d)
FIG. 3. (Color online) Tunneling through a barrier for (a), (b) MLG with $E_{F}=3 t a k_{F} / 2=81.6 \mathrm{meV}$ and (c), (d) BLG with $E_{F}=$ $\left(3 \operatorname{tak}_{F} / 2\right)^{2} / \gamma_{1}=17.1 \mathrm{meV}$. In (b), red (light gray) and blue (dark gray) curves correspond to $V_{0}=196.8 \mathrm{meV}$ and $V_{0}=280.3 \mathrm{meV}$, respectively. In (d), red (light gray) and blue (dark gray) curves correspond to $V_{0}=48.7 \mathrm{meV}$ and $V_{0}=100.7 \mathrm{meV}$, respectively. In both cases the barrier width is $D=100 \mathrm{~nm}$ and the incoming Fermi wave vector is $k_{F}=2 \pi / 50 \mathrm{~nm}^{-1}$, as considered in Ref. 22 .

only less than $2 \%$, so that the number of hexagons used here amounts to $D /(\sqrt{3} a)=4 \times[D]_{\mathrm{nm}}=400$.

The resulting transmission probabilities as a function of the incident angle $\phi$ are depicted in Figs. 3(b) and 3(d). They reproduce the results of Fig. 2 in Ref. 22 almost perfectly, if we choose slightly different $E_{F}$ and $V_{0}$, to which the transmissions at finite angles are sensitive. The remaining tiny difference between our TBM results and their Dirac theory results $^{39}$ simply reflects the basic difference between the two approaches: For graphene the effective Dirac theory is valid only for energies close to the Dirac point, while the TBM is suitable for the entire energy range.

Note that the maximal values of the transmission functions in Fig. 3 are 2, since the valley degeneracy is automatically incorporated in the tight-binding formalism. Later when we take spin also into account, the maximum of the transmission function will be 4 . The transmission probabilities calculated by the Dirac theory always have their maximum of 1 due to the normalized incoming wave, unless a proper degeneracy factor is taken into account.

\section{Klein tunneling in MLG: Sharp vs smooth interface}

Tunneling in MLG through a pn junction exhibits probability one at normal incidence and is called Klein tunneling. In experiments, a graphene $p n$ junction can be realized by using a backgate, which tunes the carrier density (and hence the Fermi level) globally, and a topgate that tunes locally the carrier density, equivalent to the potential step $V_{0}$ at the other side. ${ }^{40}$ The carrier densities on the two sides can be controlled to be of opposite signs, forming the $p n$ junction. In between, however, the variation of the carrier density is never abrupt in reality. Cheianov and Fal'ko showed, based on the Dirac theory, that the interface of the $p n$ junction actually matters. ${ }^{24}$ They considered symmetric $p n$ junctions (i.e., $V_{0}=2 E_{F}$ ) with 


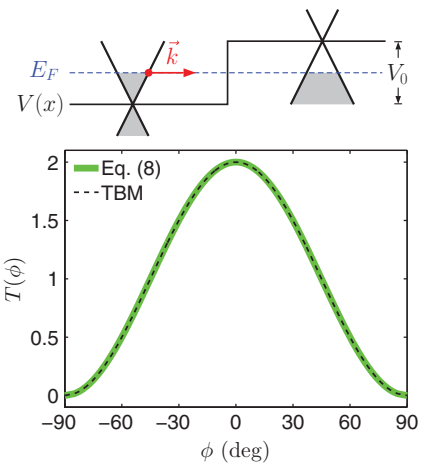

(a)
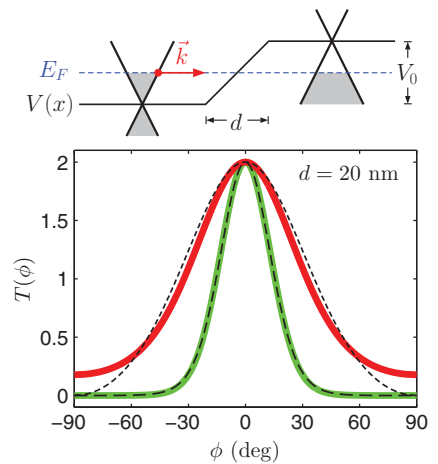

(b)
FIG. 4. (Color online) Klein tunneling in MLG through a pn junction with a (a) sharp and (b) smooth interface. (a) Comparison between TBM (dashed line) and Eq. (8) [solid green (gray)] showing perfect agreement $\left(E_{F}=80 \mathrm{meV}\right)$. (b) Comparison between TBM (long and short dashed) and Eq. (11) [solid green (light gray) and red (dark gray)] for $k_{F} d \approx 6.16\left(E_{F}=200 \mathrm{meV}\right)$ and $k_{F} d \approx 1.54$ $\left(E_{F}=50 \mathrm{meV}\right)$, respectively.

sharp and linearly smooth interfaces, which we briefly review and compare with our TBM results in the following.

a. Sharp interface. For a symmetric pn junction with a sharp interface [see the schematic in Fig. 4(a)], the transmission probability as a function of $\phi$ was written as ${ }^{24}$

$$
T(\phi)=\cos ^{2} \phi
$$

which does not depend on the potential step height. This surprisingly simple expression matches our TBM result always perfectly as long as $V_{0}=2 E_{F}$, as shown in Fig. 4(a).

For a step potential with arbitrary height $V_{0} \neq 2 E_{F}$, the transmission probability as a function of the incident angle $\phi$ and the outgoing angle $\theta$ can be derived as

$$
T(\phi, \theta)=\frac{2 \cos \phi \cos \theta}{1+\cos (\phi+\theta)},
$$

which agrees with our TBM calculation equally well as the symmetric case (not shown). The two angles $\phi$ and $\theta$ are connected to each other due to conservation of transverse momentum by

$$
\sin \theta=s \frac{\left|E_{F}\right|}{\left|E_{F}-V_{0}\right|} \sin \phi,
$$

where $s=+1$ for $n n^{\prime}$ or $p p^{\prime}$ and -1 for $n p$ or $p n$. Equation (9) clearly recovers the symmetric $p n$ junction case of Eq. (8) when choosing $s=-1$ and $V_{0}=2 E_{F}$ in Eq. (10). Note that in the case of $\left|E_{F}-V_{0}\right|<\left|E_{F}\right|$, the Fermi wave vector in the outgoing region is shorter than that in the incoming region, and an additional constraint for $\phi$ has to be applied to ensure $|\sin \theta| \leqslant 1$ [i.e., $\phi \leqslant\left|\phi_{c}\right|$ with $\left.\phi_{c}=\sin ^{-1}\left(\left|E_{F}-V_{0}\right| /\left|E_{F}\right|\right)\right]$.

Previously it has been stated that the single-valley Dirac picture, based on which Eqs. (8) and (9) are derived, is not equivalent to the TBM. ${ }^{41}$ The difference in their work, however, becomes noticeable only when the distance between one of the involved energies and the Dirac point exceeds roughly $300 \mathrm{meV}$. In our simulation, indeed the deviation for the symmetric $p n$ junction case with, say $E_{F}=300 \mathrm{meV}$, is less than $0.5 \%$. The agreement of our TBM and the Dirac theory therefore confirms that the intervalley scattering, which is mainly responsible for the nonequivalence at high energies, is indeed negligible.

b. Smooth interface. For symmetric pn junctions with a linearly varying region of width $d$ [see the schematic in Fig. 4(b)], the analytical derivation for the transmission probability within the Dirac theory yields ${ }^{24}$

$$
T(\phi)=\exp \left(-\pi \frac{k_{F} d}{2} \sin ^{2} \phi\right)
$$

for $k_{F} d \gg 1 .^{42}$ This formula, together with the validity criterion $k_{F} d \gg 1$, are tested by our tight-binding calculations shown in Fig. 4(b), where two sets of parameters are considered. For $k_{F} d \approx 6.16$ we find very good agreement with Eq. (11), while the result for $k_{F} d \approx 1.54$ exhibits noticeable deviations from the analytical prediction at large angles $|\phi|$. The smoothing function was assumed in their work as linear but the reality might be much more complicated, which is then not accessible by the Dirac theory but again straightforward by our tight-binding calculation. Nevertheless, the exponential form of Eq. (11) is still a good description regardless of the actual form of the smoothing function, as we have numerically checked. What really matters is only the product $k_{F} d$.

Unlike the sharp $p n$ interface, a compact form of transmission probability for the asymmetric case does not exist so far.

\section{B. $p n$ junction: BLG vs MLG $+R$}

We next show the direct correspondence between BLG and $\mathrm{MLG}+\mathrm{R}$ by considering exactly the same potential barrier and incident Fermi energy as in Fig. 3(d) for BLG, and set $3 t_{R}=\gamma_{1}=0.39 \mathrm{eV}$ for MLG $+\mathrm{R}$ here. (A discussion with weaker, realistic $t_{R}$ will be continued in the next section.) The total transmission shown in Fig. 5 for $M L G+R$ indeed resembles the curves in Fig. 3(d) for BLG, as expected due to the identical form of their low-energy dispersions (1) and (2). The most important feature of chiral tunneling in BLG, forbidden normal transmission, now appears also in the
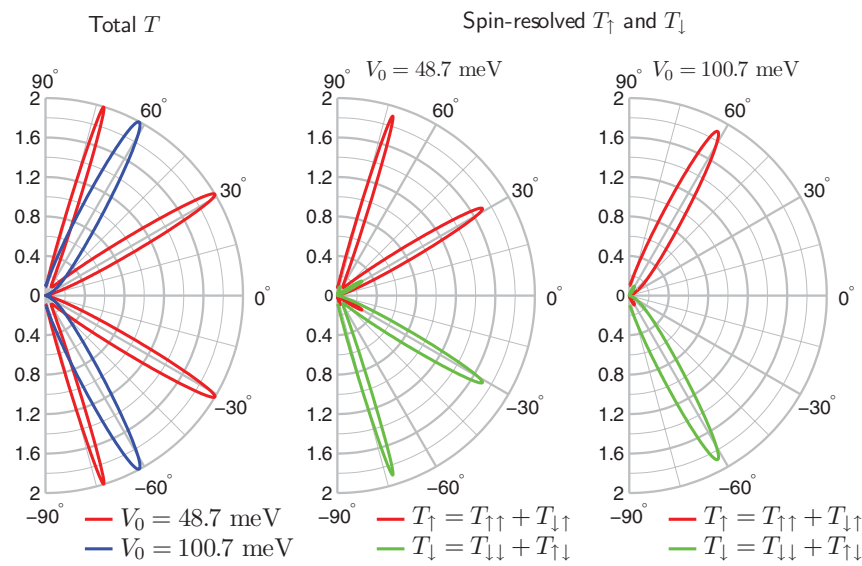

FIG. 5. (Color online) (a) Angle-resolved total transmission $T$ for tunneling through a pnp junction in $\mathrm{MLG}+\mathrm{R}$ with the same barrier height $V_{0}$, barrier width $D$, and Fermi energy $E_{F}$ as used in Fig. 3(d) for BLG, and a substitution $3 t_{R}=\gamma_{1}=0.39 \mathrm{eV}$. (b) and (c) show spin-resolved transmission probabilities for $V_{0}=48.7 \mathrm{meV}$ and $V_{0}=100.7 \mathrm{meV}$, respectively. 
case of MLG + R. In BLG, $T(\phi=0)=0$ was understood as the consequence of pseudospin conservation. For MLG $+\mathrm{R}$, $T(\phi=0)=0$ can be expected as the consequence of real-spin conservation. Indeed, this can be demonstrated by computing the nonequilibrium local spin density, which can be obtained from the lesser Green's function, ${ }^{43}$ considering two cases, $0<$ $E_{F}<3 t_{R}$ and $-3 t_{R}<E_{F}<0$, both with $k_{y}=0$. Within this single-band transmission, the local spin densities for positive and negative $E_{F}$ point to opposite directions, indicating that normal incidence transmission between $n$ and $p$ regions will be forbidden.

Next we discuss the spin-resolved transmission. The quantization axis is chosen as the out-of-plane direction, so that the transmission of, for example, $T_{\downarrow \uparrow}$ means the probability of an incoming $+S_{z}$ electron ending up as an outgoing $-S_{z}$ one. Since the incoming angle dependence $\phi$ of the transmission probabilities are analyzed, we define $T_{\uparrow}=T_{\uparrow \uparrow}+T_{\downarrow \uparrow}$ as the transmission ability of the $+S_{z}$ electron (or $\uparrow$ spin), and vice versa. (Alternatively, one can also analyze the outgoing angle dependence and define $T_{\uparrow}$ as $T_{\uparrow \uparrow}+T_{\uparrow \downarrow}$, not used here. Either way, the total transmission $\sum_{\sigma, \sigma^{\prime}=\uparrow, \downarrow} T_{\sigma \sigma^{\prime}}=T_{\uparrow}+T_{\downarrow}=T$ is ensured.)

The choice of quantization axis $z$ is not necessary but facilitates relating the present spin-dependent tunneling in MLG with the issue of intrinsic spin-Hall effect previously discussed in semiconductors. The spin-resolved transmission curves shown in Fig. 5 exhibit opposite lateral preference of the $\uparrow$ and $\downarrow$ electron spins, which is an intrinsic spin-Hall mechanism due to the Rashba SOC. In a semiconductor two-dimensional electron gas (i.e., a continuous system rather than discrete as in the TBM), such an intrinsic spin-Hall deflection of opposite $S_{z}$ electrons can be easily explained by the concept of a spin-orbit force based on the Heisenberg equation of motion, ${ }^{44,45}$

$$
\mathbf{F}_{\text {so }}=\frac{m}{i \hbar}\left[\frac{1}{i \hbar}[\mathbf{r}, \mathcal{H}], \mathcal{H}\right]=\frac{2 m \alpha_{R}^{2}}{\hbar^{3}}\left(\mathbf{p} \times \mathbf{e}_{z}\right) \sigma^{z} .
$$

Here $\mathcal{H}=p^{2} / 2 m+\left(\alpha_{R} / \hbar\right)\left(p_{y} \sigma^{x}-p_{x} \sigma^{y}\right)$ is the continuous two-dimensional Hamiltonian with Rashba SOC, $\mathbf{r}$ and $\mathbf{p}$ are the position and momentum operators, $\alpha_{R}$ is the Rashba coupling parameter (rather than the hopping one, $t_{R}$ ), and $\sigma^{z}$ is the sign of the $S_{z}$ spin component. The $T_{\uparrow}$ and $T_{\downarrow}$ curves shown in Fig. 5 therefore reveal a combined effect of forbidden normal transmission due to conservation of real spin and the intrinsic spin-Hall deflection that can be understood by Eq. (12).

A few remarks are due before we move on. To connect BLG with $\mathrm{MLG}+\mathrm{R}$ we put $3 t_{R}=\gamma_{1}=0.39 \mathrm{eV}$, which is apparently far from reality. In general the Rashba splitting induced by electrical gating is roughly of or less than the order of $100 \mu \mathrm{eV}$ (see Sec. IV). Fermi energy lying within this splitting, which is also our main interest, projects to a much shorter Fermi wave vector $k_{F}$, leading to a much longer $d$ up to a few or a few tens of microns in order for $k_{F} d \gg 1$ to be valid. This implies that the influence of the interface on the tunneling in $\mathrm{MLG}+\mathrm{R}$ is normally negligible, unless $d$ is that long. In addition, tunneling through a pnp junction will also require a long barrier width $D$ for electrons subject to such a short $k_{F}$; otherwise, the barrier is merely a weak perturbation to the electron due to its long Fermi wave length. Based on these
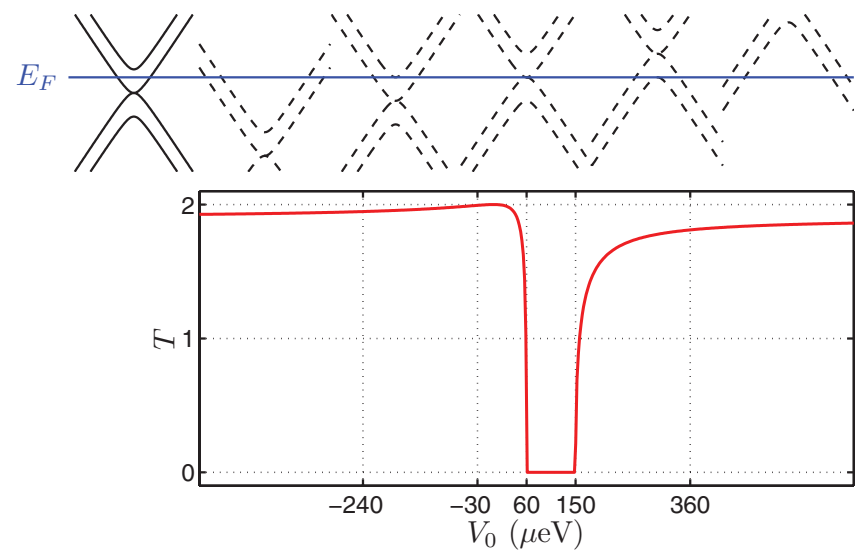

FIG. 6. (Color online) Transmission $T$ at normal incidence $\left(k_{y}=\right.$ $0)$ as a function of potential step height $V_{0}$ for tunneling through a $p n$ junction in MLG $+\mathrm{R}$. The leftmost solid band diagram above the main panel corresponds to the incoming $n$ side. The five ticks on the $V_{0}$ axis correspond to the above five dashed band diagrams for the outgoing side.

remarks, we will focus in the next section only on $p n$ junctions in $\mathrm{MLG}+\mathrm{R}$ with a reasonable Rashba hopping parameter.

\section{C. $p n$ junction in MLG $+R$}

In the following we demonstrate in detail the role of Rashba SOC in tunneling through a potential step in MLG $+\mathrm{R}$. The Rashba hopping parameter will be fixed to $t_{R}=30 \mu \mathrm{eV}$ and the Fermi energy in most cases to $E_{F}=2 t_{R}$, which lies within the spin-orbit splitting $3 t_{R}$ (see Fig. 1).

\section{Normal incidence}

We begin with the case of normal incidence, $k_{y}=0$. In Sec. III B we have discussed the one-band transmission selection rule (i.e., $n \leftrightarrow p$ transmission is forbidden). The transmission from the left side at Fermi energy $0<E_{F}<3 t_{R}$ to the right side with potential $V_{0}$ is expected to be zero whenever a single-band $n \rightarrow p$ transmission is attempted. Indeed, as shown in Fig. 6, a zero transmission gap of $T$ as a function of $V_{0}$ is found. The gap lies in the interval of $E_{F}<V_{0}<E_{F}+3 t_{R}$, corresponding to the single-band $n \rightarrow p$ transmission. Note that contrary to the valley-valve effect in zigzag nanoribbons, ${ }^{46-48}$ the gap shown here arises solely due to a bulk property.

\section{Angle- and spin-resolved transmission}

We proceed with angle- and spin-resolved transmission and consider first the trivial case with $E_{F}=0.5 \mathrm{meV}$ well above the Rashba splitting $3 t_{R}=90 \mu \mathrm{eV}$, as shown in Fig. 7 . In this case the maximum of $T=T_{\uparrow}+T_{\downarrow}$ is 4 since two spin subbands and two valleys are involved in transport. The total transmission curve resembles the expected $\cos ^{2} \phi$ behavior as discussed in Sec. III A 2, showing that the Rashba effect plays only a minor role. The spin-resolved $T_{\uparrow}$ and $T_{\downarrow}$ curves differ only slightly at $|\phi|=\sin ^{-1}\left(k_{F}^{\text {in }} / k_{F}^{\text {out }}\right) \approx 56^{\circ}$, where $k_{F}^{\text {in }}$ and $k_{F}^{\text {out }}$ are the inner and outer radius of the two concentric Fermi circles, respectively. Tunneling in BLG with $E_{F}$ well above $\gamma_{1}$ behaves similarly (i.e., the interlayer 

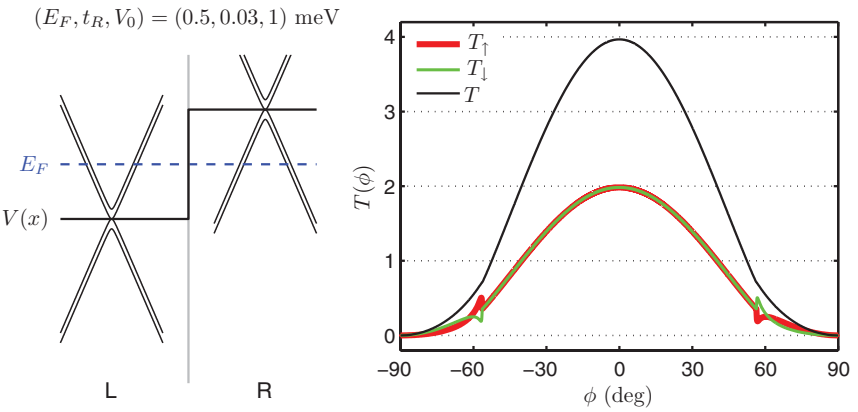

FIG. 7. (Color online) Angular dependence of total $(T)$ and spinresolved $\left(T_{\uparrow}\right.$ and $\left.T_{\downarrow}\right)$ transmissions for $E_{F}=0.5 \mathrm{meV}$ well above the Rashba splitting $3 t_{R}=90 \mu \mathrm{eV}$.

coupling $\gamma_{1}$ in BLG no longer plays an important role in the process of chiral tunneling when the transport occurs at $E_{F} \gg \gamma_{1}$ ), as we have numerically checked. In other words, the chiral tunneling in BLG with $E_{F} \gg \gamma_{1}$ and in MLG + R with $E_{F} \gg 3 t_{R}$ recovers the Klein tunneling behavior as in MLG.

Of particular interest is the nontrivial case with $\left|E_{F}\right|<3 t_{R}$. As a test, we first consider $V_{0}=0$ as shown in Fig. 8(a). In the absence of the potential step, the total transmission function $T$ reaches its maximum of 2 (one spin subband times valley degeneracy of two) for any angle $\phi$, as it should. The opposite lateral deflection tendency of the $\uparrow$ and $\downarrow$ spins is again clearly seen and can be explained based on Eq. (12) as discussed in Sec. III B.

The most important case is that of Fermi energy $E_{F} \in$ $\left(0,3 t_{R}\right)$ and potential height $V_{0} \in\left(E_{F}, E_{F}+3 t_{R}\right)$. A specific example with $V_{0}=100 \mu \mathrm{eV}$ is shown in Fig. 8(b), which exhibits the combined effect of the forbidden normal transmission $[T(\phi=0)=0]$ and spin-Hall deflection. The number of high transmission peaks is always two. ${ }^{49}$ Compared to the previous trivial case $\left(E_{F}>3 t_{R}\right.$; Fig. 7) where $T_{\uparrow}$ and $T_{\downarrow}$ do not significantly differ, the separation of the opposite $\uparrow$ and $\downarrow$ spins is distinctly enhanced. Whether this could

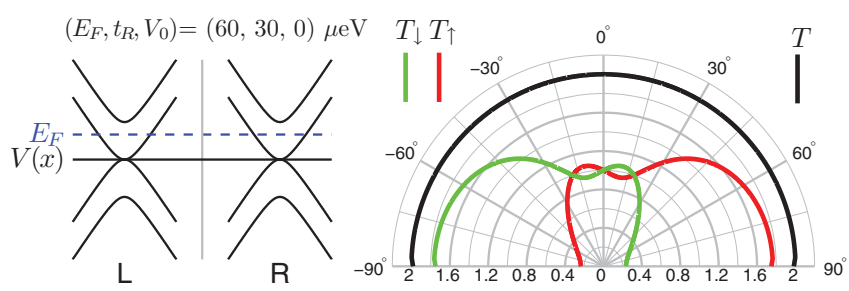

(a)

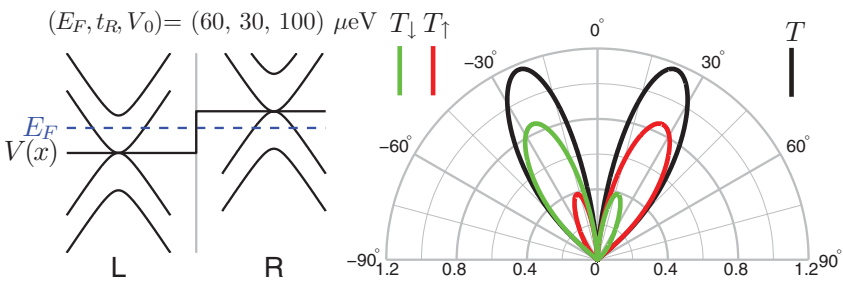

(b)

FIG. 8. (Color online) Angular dependence of total and spinresolved transmissions through a $p n$ junction in MLG $+\mathrm{R}$ with (a) zero potential and (b) finite potential. Parameters used are given above. be a new type of intrinsic spin-Hall mechanism in graphene deserves a further investigation, and is left as a possible future direction.

We summarize the discussion of angle- and spin-resolved transmission by mapping $T\left(\phi, V_{0}\right)$ in Fig. 9. Four different transport regimes can be identified:

(1) $V_{0}<0$, single $n$ band to single/multiple $n$ band(s) transmission regime.

(2) $0<V_{0}<E_{F}$, single $n$ band to single $n$ band transmission regime; distinct spin-resolved $T_{\uparrow}$ and $T_{\downarrow}$, and high total $T$ limited by a critical angle $\phi_{c}=\sin ^{-1}\left(\left|E_{F}-V_{0}\right| /\left|E_{F}\right|\right)$.

(3) $E_{F}<V_{0}<E_{F}+3 t_{R}$, single $n$ band to single $p$ band transmission regime; combined effects of forbidden normal transmission and spin-Hall deflection.

(4) $V_{0}>E_{F}+3 t_{R}$, single $n$ band to multiple $p$ bands transmission regime.

Note that a vertical scan in Fig. 9 at $\phi=0$ corresponds to Fig. 6, and horizontal scans at $V_{0}=0$ and $V_{0}=100 \mu \mathrm{eV}$ to Figs. 8(a) and 8(b), respectively. These four regimes will be helpful in the following discussion of conductance.

\section{Integrated conductance}

Finally, we calculate the conductance of the $p n$ junction in MLG $+\mathrm{R}$ by integrating $T(\phi)$, or equivalently, $T\left(k_{y}\right)$, with respect to the transverse Bloch momentum,

$$
G=\frac{e^{2} / h}{2 k_{F}} \int_{-k_{F}}^{k_{F}} T\left(k_{y}\right) d k_{y},
$$

where the prefactor ensures the maximal value of the Landauer-Büttiker-type ballistic conductance to be $e^{2} / h$ times the maximal number of modes. ${ }^{37}$ We compare the conductance of the $p n$ junction in MLG $\left(t_{R}=0\right)$ and in $\mathrm{MLG}+\mathrm{R}\left(t_{R}=\right.$ $30 \mu \mathrm{eV})$ as a function of the potential step height $V_{0}$, as shown in Fig. 10. Since the Fermi level is fixed to $E_{F}=60 \mu \mathrm{eV}$ for both cases, the transport for $t_{R}=0$ will involve two spin and two valley degeneracies, leading to the maximal $G$ of $4 e^{2} / h$, while in the case of $t_{R}=30 \mu \mathrm{eV}$ only one spin subband is projected, leading to the maximal $G$ of $2 e^{2} / h$. The maximal $G$ occurs always at $V_{0}=0$ that corresponds to an ungated clean bulk graphene. Zero conductance, on the other hand, occurs at $V_{0}=E_{F}$ since no states at the outgoing region are available at this charge neutrality point.

Different transmission regimes can be distinguished based on our previous discussion for Fig. 9. For $V_{0} \in[0,60] \mu \mathrm{eV}$ ( $n \rightarrow n$ transmission), the rise of $V_{0}$ shrinks the Fermi circle at the outgoing region and hence introduces a critical transverse momentum, outside which the transmission is suppressed due to the lack of outgoing states. The critical transverse momentum reduces linearly with $V_{0}$ for MLG due to the linear dispersion. The conductance $G$, Eq. (13), therefore reduces also linearly with $V_{0}$. In the presence of the Rashba SOC, the low-energy dispersion becomes quadratic, and so does the reduction of $G$ with $V_{0}$ in MLG + R.

For $V_{0} \in[60,150] \mu \mathrm{eV}(n \rightarrow p$ transmission $)$, the conductance of MLG rises faster than that of MLG + R, possibly due to the help of Klein tunneling. At $V_{0}=150 \mu \mathrm{eV}$, a sudden jump (or a shoulder) occurs in the case of $\mathrm{MLG}+\mathrm{R}$ since the second spin subband at the outgoing region starts to participate in transport. This jump does not occur in the MLG case 

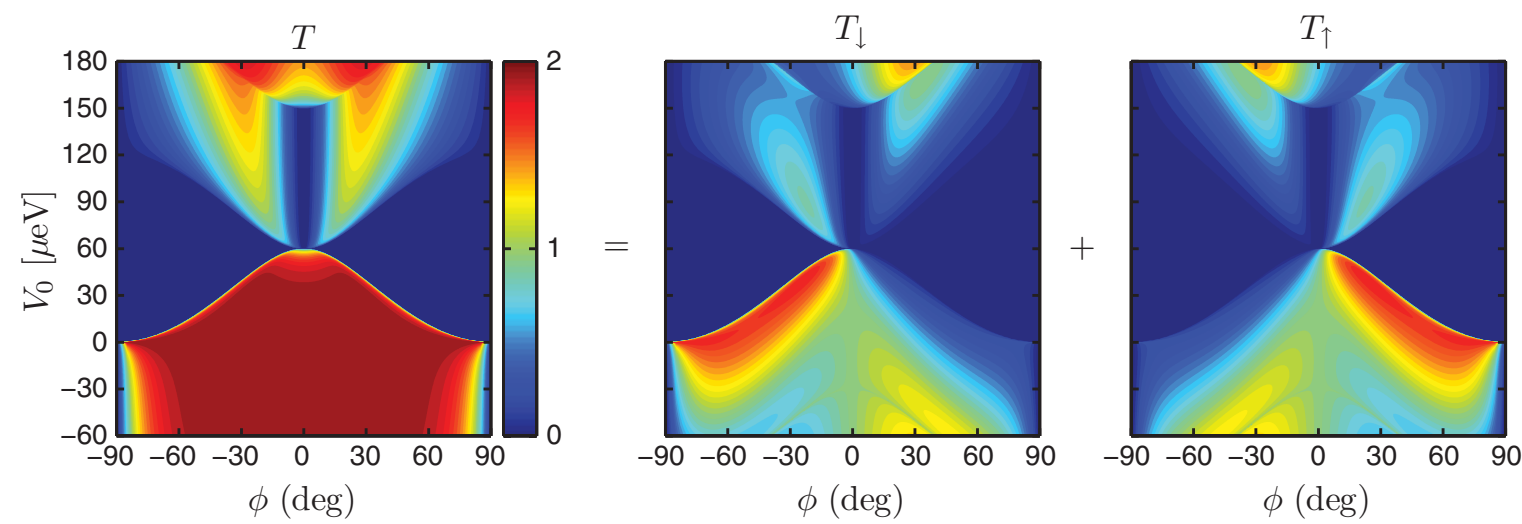

FIG. 9. (Color online) Transmission through a $p n$ junction in MLG $+\mathrm{R}$ as a function of incident angle $\phi$ and potential step height $V_{0}$. Four transmission regimes can be distinguished: (i) $V_{0}<0$, (ii) $0<V_{0}<E_{F}$, (iii) $E_{F}<V_{0}<E_{F}+3 t_{R}$, and (iv) $V_{0}>E_{F}+3 t_{R}$, with $E_{F}=60 \mu \mathrm{eV}$ and $3 t_{R}=90 \mu \mathrm{eV}$.

since both spin subbands are always degenerate. An earlier related work based on Dirac theory considered both intrinsic and Rashba SOCs. ${ }^{18}$ The $V_{0}$ dependence of $G$ for the Rashba dominated case in that work agrees well with the MLG $+\mathrm{R}$ curve shown in Fig. 10, including the shoulder.

\section{EXPERIMENTAL ASPECTS}

\section{A. Rashba spin splitting in graphene}

Whereas the Rashba spin splitting in MLG induced by an applied electric field is in general in the order of no more than $100 \mu \mathrm{eV}$, which is beyond the present resolution of angle-resolved photoelectron spectroscopy (ARPES), direct experimental observation of the Rashba spin splitting at $K$ and $K^{\prime}$ in agreement with the first-principles calculations ${ }^{9,10}$ is so far not reported. An earlier experiment on epitaxial graphene layers on a Ni(111) surface reported a large Rashba interaction $^{50}$ up to $225 \mathrm{meV}$ but was soon questioned since the splitting might simply reveal a Zeeman-type splitting due to the ferromagnetic nature of nickel. ${ }^{51}$ An intercalated $\mathrm{Au}$ monolayer between the graphene layer and the $\mathrm{Ni}(111)$ substrate reduced the splitting to about $13 \mathrm{meV}$ and was concluded as the Rashba effect on the $\pi$ states supported by spin-resolved ARPES. ${ }^{52}$ However, the low-energy band structure of $M L G+R$ at that time was not yet clear, and a simplified picture was adopted in the explanation of the

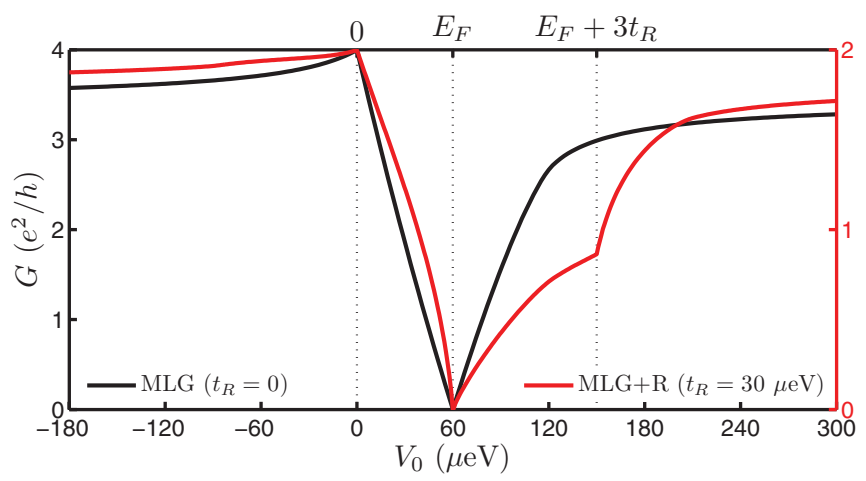

FIG. 10. (Color online) Integrated conductance of the ballistic $p n$ junction in MLG with $t_{R}=0$ and MLG $+\mathrm{R}$ with $t_{R}=30 \mu \mathrm{eV}$. measured spin splitting. In addition, transport properties of graphene based on metallic substrates can be difficult to isolate since a large bulk current will interfere as background. ${ }^{53}$

Throughout the above calculations we have mostly focused on a rather weak Rashba hopping parameter $t_{R}=30 \mu \mathrm{eV}$, yielding a splitting at the $K$ and $K^{\prime}$ points $3 t_{R}=90 \mu \mathrm{eV}$, which is a realistic and rather conservative estimate for the gate-voltage-induced Rashba SOC strength. A recent proposal of impurity-induced SOC in graphene, ${ }^{54}$ however, indicated that the coupling strength can be strongly enhanced by putting heavy adatoms ${ }^{55}$ as well as by hydrogenation. ${ }^{54,56}$

\section{B. Klein tunneling in MLG}

Indirect and direct experimental evidences of Klein tunneling in MLG have been reported recently. ${ }^{57,58}$ For detailed reviews, we refer to Refs. 2,3,20,21 and 59. A very recent experiment on transport through a pn junction in MLG used an embedded local gate, which yields high quality ballistic transport and perfectly independent control of the local carrier density, as well as the feature of Klein tunneling. ${ }^{60}$

Recall the $t_{R}=0$ curve of conductance for MLG shown in Fig. 10. Overall, the conductance for $n \rightarrow n$ transmission with $V_{0}<0$ is always higher than that for $n \rightarrow p$ transmission with $V_{0}>E_{F}$. Even though Klein tunneling leads to perfect transmission at normal incidence in the latter case, the decay of $T$ with incident angle eventually yields a lower conductance after integration. This feature has been agreed in recent experiments for $p n$ and pnp junctions in MLG. ${ }^{40,57,58,60-64}$ The difference of the conductance, or equivalently the resistance, between the $n n$ and $n p$ (or between $p p$ and $p n$ ) in experiments is even more obvious possibly due to the smooth interface that leads to an exponentially decaying form of $T,{ }^{24}$ as we have reviewed and discussed in Sec. III A 2. In fact, for MLG we have numerically checked $G$ for $p n$ junctions with a smooth interface, which indeed can enhance the difference of $G$ between the $n n$ and $n p$ regimes.

Another interesting feature so far experimentally reported only in Refs. 58 and 60 is the Fabry-Perot oscillation of the conductance for $p n p$ junctions due to the interference between the two interfaces of the central barrier. This feature requires the system to be ballistic and can be naturally revealed by our 
tight-binding transport calculation, which we will elaborate elsewhere in the future.

\section{CONCLUSION AND OUTLOOK}

In conclusion, we have employed tight-binding calculations to show that transport properties of MLG + R behave as BLG due to their identical form of the low-energy dispersion, choosing the chiral tunneling in $p n$ and $p n p$ junctions as a concrete example. Within single-band transmission, normal incidence transmission through a $p n$ junction in BLG with $\left|E_{F}\right|<\gamma_{1}$ is forbidden as a consequence of pseudospin conservation, ${ }^{22}$ while in MLG $+\mathrm{R}$ with $\left|E_{F}\right|<3 t_{R}$ this forbidden transmission also occurs but as a consequence of real-spin conservation. In mapping the angle- and spin-resolved transmission for the $M L G+R$ case, a combined effect of forbidden normal transmission and intrinsic spin-Hall deflection is revealed [Fig. 8(b)]. Compared to the potential-free spin-Hall deflection case as shown in Fig. 8(a), where $T_{\uparrow}=T_{\downarrow}=1$ at $\phi=0$, the effect of the $p n$ junction seems to force the up and down spins to separate since $T_{\uparrow}=T_{\downarrow}=0$ at $\phi=0$. The feature revealed in Fig. 8(b) may therefore suggest a new type of intrinsic spin-Hall mechanism in MLG.

Within multiband transmission, however, the Rashba SOC in MLG no longer plays an important role when $\left|E_{F}\right| \gg 3 t_{R}$ (Fig. 7). Likewise, the interlayer hopping $\gamma_{1}$ in BLG becomes unimportant when $\left|E_{F}\right| \gg \gamma_{1}$. Transport in both MLG $+\mathrm{R}$ with $\left|E_{F}\right| \gg 3 t_{R}$ and BLG with $\left|E_{F}\right| \gg \gamma_{1}$ recovers to that in MLG, despite the usually very different energy scales of $3 t_{R}$ and $\gamma_{1}$. In view of the distinct transmission patterns in $\mathrm{MLG}+\mathrm{R}$ with $\left|E_{F}\right|<3 t_{R}$ [Fig. 8(b)] and $\left|E_{F}\right| \gg 3 t_{R}$ (Fig. 7), as an interesting conjecture for the BLG case one expects very different scattering regimes for $\left|E_{F}\right|<\gamma_{1}$ and $\left|E_{F}\right| \gg \gamma_{1}$. The former is well discussed in the literature and exhibits strong scattering [Fig. 3(d)] while the latter is less discussed and the scattering is expected to be strongly suppressed.

MLG and BLG are known to behave quite differently in general, in the sense of single-band transmission. Whereas turning MLG directly into BLG is in principle not possible, steering MLG to MLG + R can be achieved simply by gating, and therefore the effect of Rashba SOC provides a possibility to continuously change the MLG-like transport properties to BLG-like properties. We expect further transport properties to behave similarly in BLG and in MLG $+\mathrm{R}$, such as the quantum Hall effect, ${ }^{65}$ as was also noted by Rashba. ${ }^{14}$

\section{ACKNOWLEDGMENTS}

We gratefully acknowledge Alexander von Humboldt Foundation (M.H.L.) and Deutsche Forschungsgemeinschaft (within SFB689) (J.B. and K.R.) for financial support.
${ }^{1}$ K. S. Novoselov, A. K. Geim, S. V. Morozov, D. Jiang, Y. Zhang, S. V. Dubonos, I. V. Grigorieva, and A. A. Firsov, Science 306, 666 (2004).

${ }^{2}$ A. H. Castro Neto, F. Guinea, N. M. R. Peres, K. S. Novoselov, and A. K. Geim, Rev. Mod. Phys. 81, 109 (2009).

${ }^{3}$ S. Das Sarma, S. Adam, E. H. Hwang, and E. Rossi, Rev. Mod. Phys. 83, 407 (2011).

${ }^{4}$ D. D. Awschalom, D. Loss, and N. Samarth (eds.), Semiconductor Spintronics and Quantum Computation (Springer, Berlin, 2002).

${ }^{5}$ I. Žutić, J. Fabian, and S. Das Sarma, Rev. Mod. Phys. 76, 323 (2004).

${ }^{6}$ A. K. Geim and K. S. Novoselov, Nat. Mater. 6, 183 (2007).

${ }^{7}$ C. L. Kane and E. J. Mele, Phys. Rev. Lett. 95, 226801 (2005).

${ }^{8}$ H. Min, J. E. Hill, N. A. Sinitsyn, B. R. Sahu, L. Kleinman, and A. H. MacDonald, Phys. Rev. B 74, 165310 (2006).

${ }^{9}$ M. Gmitra, S. Konschuh, C. Ertler, C. Ambrosch-Draxl, and J. Fabian, Phys. Rev. B 80, 235431 (2009).

${ }^{10} \mathrm{~S}$. Abdelouahed, A. Ernst, J. Henk, I. V. Maznichenko, and I. Mertig, Phys. Rev. B 82, 125424 (2010).

${ }^{11}$ F. D. M. Haldane, Phys. Rev. Lett. 61, 2015 (1988).

${ }^{12}$ E. I. Rashba, Sov. Phys. Solid State 2, 1109 (1960).

${ }^{13}$ Y. A. Bychkov and E. I. Rashba, JETP Lett. 39, 78 (1984).

${ }^{14}$ E. I. Rashba, Phys. Rev. B 79, 161409(R) (2009).

${ }^{15}$ M.-H. Liu and C.-R. Chang, Phys. Rev. B 80, 241304(R) (2009).

${ }^{16}$ Due to a minor difference in the definition of the Rashba coupling in the tight-binding Hamiltonian, the splitting $3 t_{R}$ here corresponds, for example, to $\lambda$ in Ref. 14 and to $2 \lambda_{R}$ in Ref. 9.

${ }^{17}$ E. McCann and V. I. Fal'ko, Phys. Rev. Lett. 96, 086805 (2006).
${ }^{18}$ A. Yamakage, K. I. Imura, J. Cayssol, and Y. Kuramoto, Europhys. Lett. 87, 47005 (2009).

${ }^{19}$ P. Rakyta, A. Kormányos, and J. Cserti, Phys. Rev. B 82, 113405 (2010).

${ }^{20}$ C. W. J. Beenakker, Rev. Mod. Phys. 80, 1337 (2008).

${ }^{21}$ P. Allain and J. Fuchs, Eur. Phys. J. B 83, 301 (2011).

${ }^{22}$ M. I. Katsnelson, K. S. Novoselov, and A. K. Geim, Nature Physics 2, 620 (2006).

${ }^{23}$ O. Klein, Z. Phys. 53, 157 (1929).

${ }^{24}$ V. V. Cheianov and V. I. Fal'ko, Phys. Rev. B 74, 041403 (2006).

${ }^{25}$ L. M. Zhang and M. M. Fogler, Phys. Rev. Lett. 100, 116804 (2008).

${ }^{26}$ E. B. Sonin, Phys. Rev. B 79, 195438 (2009).

${ }^{27}$ C. Bai, Y. Yang, and X. Zhang, Physica E: Low-dimensional Systems and Nanostructures 42, 1431 (2010).

${ }^{28}$ E. Rossi, J. H. Bardarson, P. W. Brouwer, and S. Das Sarma, Phys. Rev. B 81, 121408 (2010).

${ }^{29}$ J. M. Pereira Jr, F. M. Peeters, A. Chaves, and G. A. Farias, Semicond. Sci. Technol. 25, 033002 (2010).

${ }^{30}$ T. Tudorovskiy, K. J. A. Reijnders, and M. I. Katsnelson, e-print arXiv:1106.3042 (to be published).

${ }^{31}$ D. Bercioux and A. De Martino, Phys. Rev. B 81, 165410 (2010).

${ }^{32}$ C. Bai, J. Wang, J. Tian, and Y. Yang, Physica E: Low-dimensional Systems and Nanostructures 43, 207 (2010).

${ }^{33}$ M. Rataj and J. Barnaś, Appl. Phys. Lett. 99, 162107 (2011).

${ }^{34}$ J. Schelter, D. Bohr, and B. Trauzettel, Phys. Rev. B 81, 195441 (2010).

${ }^{35}$ S. Konschuh, M. Gmitra, and J. Fabian, Phys. Rev. B 82, 245412 (2010).

${ }^{36}$ M. Wimmer, Ph.D. thesis, Universität Regensburg, 2008. 
${ }^{37}$ S. Datta, Electronic Transport in Mesoscopic Systems (Cambridge University Press, Cambridge, 1995).

${ }^{38}$ B. K. Nikolić and R. L. Dragomirova, Semicond. Sci. Technol. 24, 064006 (2009).

${ }^{39}$ The unity transmission peaks (except the $0^{\circ}$ peaks for MLG) are shifted by less than $3^{\circ}$ compared to Fig. 2 of Ref. 22.

${ }^{40}$ J. R. Williams, L. DiCarlo, and C. M. Marcus, Science 317, 638 (2007).

${ }^{41}$ C. Tang, Y. Zheng, G. Li, and L. Li, Solid State Commun. 148, 455 (2008).

${ }^{42}$ Note that an additional factor of $1 / 2$ in the exponent of Eq. (11) as compared to the original formula given in Ref. 24 comes from the fact that the linear potential profile across the interface changes from $-V_{0}$ to $V_{0}$ in Ref. 24, but here from 0 to $V_{0}$, that is, $k_{F}$ reduces to $k_{F} / 2$.

${ }^{43}$ B. K. Nikolić, L. P. Zarbo, and S. Souma, Phys. Rev. B 73, 075303 (2006).

${ }^{44}$ J. Li, L. Hu, and S.-Q. Shen, Phys. Rev. B 71, 241305 (2005).

${ }^{45}$ B. K. Nikolić, L. P. Zârbo, and S. Welack, Phys. Rev. B 72, 075335 (2005).

${ }^{46}$ K. Wakabayashi and T. Aoki, Int. J. Mod. Phys. B 16, 4897 (2002).

${ }^{47}$ A. Rycerz, J. Tworzydlo, and C. W. J. Beenakker, Nat. Phys. 3, 172 (2007).

${ }^{48}$ A. Cresti, G. Grosso, and G. P. Parravicini, Phys. Rev. B 77, 233402 (2008).

${ }^{49} \mathrm{We}$ have numerically checked that the double-peak feature of $T_{\mathrm{tot}}$ in the single-band $n \leftrightarrow p$ transmission regime shown in Fig. 8(b) still holds even if the intrinsic SOC is present, as long as the Rashba coupling dominates.

${ }^{50}$ Y. S. Dedkov, M. Fonin, U. Rüdiger, and C. Laubschat, Phys. Rev. Lett. 100, 107602 (2008).

${ }^{51}$ O. Rader, A. Varykhalov, J. Sánchez-Barriga, D. Marchenko, A. Rybkin, and A. M. Shikin, Phys. Rev. Lett. 102, 057602 (2009).
${ }^{52}$ A. Varykhalov, J. Sanchez-Barriga, A. M. Shikin, C. Biswas, E. Vescovo, A. Rybkin, D. Marchenko, and O. Rader, Phys. Rev. Lett. 101, 157601 (2008)

${ }^{53}$ K. Yaji, Y. Ohtsubo, S. Hatta, H. Okuyama, K. Miyamoto, T. Okuda, A. Kimura, H. Namatame, M. Taniguchi, and T. Aruga, Nat. Comm. 1, 17 (2010).

${ }^{54}$ A. H. Castro Neto and F. Guinea, Phys. Rev. Lett. 103, 026804 (2009).

${ }^{55}$ C. Weeks, J. Hu, J. Alicea, M. Franz, and R. Wu, Phys. Rev. X 1, 021001 (2011)

${ }^{56}$ D. C. Elias, R. R. Nair, T. M. G. Mohiuddin, S. V. Morozov, P. Blake, M. P. Halsall, A. C. Ferrari, D. W. Boukhvalov, M. I. Katsnelson, A. K. Geim, and K. S. Novoselov, Science 323, 610 (2009).

${ }^{57}$ N. Stander, B. Huard, and D. Goldhaber-Gordon, Phys. Rev. Lett. 102, 026807 (2009).

${ }^{58}$ A. F. Young and P. Kim, Nat. Phys. 5, 222 (2009).

${ }^{59}$ A. F. Young and P. Kim, Annual Review of Condensed Matter Physics 2, 101 (2011).

${ }^{60}$ S.-G. Nam, D.-K. Ki, J. W. Park, Y. Kim, J. S. Kim, and H.-J. Lee, Nanotechnology 22, 415203 (2011).

${ }^{61}$ B. Huard, J. A. Sulpizio, N. Stander, K. Todd, B. Yang, and D. Goldhaber-Gordon, Phys. Rev. Lett. 98, 236803 (2007).

${ }^{62}$ B. Özyilmaz, P. Jarillo-Herrero, D. Efetov, D. A. Abanin, L. S. Levitov, and P. Kim, Phys. Rev. Lett. 99, 166804 (2007).

${ }^{63}$ G. Liu, J. Velasco, Jr., W. Bao, and C. N. Lau, Appl. Phys. Lett. 92, 203103 (2008)

${ }^{64}$ N. M. Gabor, J. C. W. Song, Q. Ma, N. L. Nair, T. Taychatanapat, K. Watanabe, T. Taniguchi, L. S. Levitov, and P. Jarillo-Herrero, Science 334, 648 (2011).

${ }^{65}$ K. S. Novoselov, A. K. Geim, S. V. Morozov, D. Jiang, M. I. Katsnelson, I. V. Grigorieva, S. V. Dubonos, and A. A. Firsov, Nature (London) 438, 197 (2005). 